\section{GRP-040 CLINICAL SIGNIFICANCE OF RECOMMENDATIONS MADE BY PHARMACISTS ABOUT DRUG-RELATED PROBLEMS (DRP)}

doi:10.1136/ejhpharm-2013-000276.040

M Buajordet. Central Norway Hospital Pharmacy Trust, The Hospital Pharmacy of Trondheim, Trondheim, Norway

Background The Ministry of Health in Norway has requested an expanded contribution from clinical pharmacy in healthcare delivery because of serious medication-related issues. Examples of this are participation in treatment teams in hospital wards and review of the patient's total use of medicine in cooperation with a medical practitioner. The concept of integrated medicines management (IMM) has been approved as a model to enhance medication effectiveness and safety.

Purpose The objective of this study was to evaluate the clinical significance of recommendations made by pharmacists in drugrelated problems (DRP).

Materials and Methods The study was conducted on a respiratory ward and a rheumatology ward at the University Hospital of St. Olav, Trondheim, Norway. Patients admitted to hospital in the period of June to October 2011 were included. All patients using one or more drugs at admission, having DRPs identified by the pharmacist according to the IMM (Integrated Medicine Management) model, were included. DRPs were identified through medicines reconciliation and medication reviews. All recommendations made by the pharmacists were independently assessed and scored by a physician with a special interest in pulmonary diseases, or respectively rheumatology, a clinical pharmacologist and a clinical pharmacist. A Hatoum six-point scoring system [1] for assessing the quality of pharmacists' interventions was used, with rankings between 1 . Adverse significance - (the recommendation supplied by the pharmacist may lead to adverse outcome and 6. Extremely significant information qualified by life and death situation.).

Results A total of 112 recommendations in 46 patients (average age 66 years), were assessed. On average 4 DRPs per patient were found. $85 \%$ of the recommendations were assessed as somewhat significant or more ( $\geq$ rank 3 ). The physicians accepted $71 \%$ of the pharmacists' recommendations.

Conclusions Recommendations made by pharmacists were assessed as clinically significant to a large extent. The fact that the physicians followed the pharmacists recommendations in most cases, demonstrates the effectiveness and value of the IMM model in improving patient drug treatment.

Reference

1. Hatoum HT et al, Evaluation of the contribution of clinical pharmacists: inpatient care and cost reduction. Drug Intelligence and Clinical Pharmacy, March 1988, Vol. 22, 252-258.

No conflict of interest.

\section{GRP-041 COLLECTION AND ANALYSIS OF ADVERSE EFFECTS AND CO-MEDICATIONS FOR OUTPATIENTS RECEIVING BOCEPREVIR- OR TELAPREVIR-BASED TREATMENT FOR CHRONIC HEPATITIS C}

doi:10.1136/ejhpharm-2013-000276.041

N Carré, G Lemaître, N Abbas, C Guérin. Hôpital Cochin, Pharmacy, Paris Cedex 14, France

Background The current treatment of chronic genotype 1 hepatitis $C$ virus infection is the triple combination of peginterferon, ribavirin and a new direct-acting antiviral (DAA), either telaprevir (TVR) or boceprevir (BOC). Potential adverse drug reactions (ADRs) represent an important problem in patient safety. In addition, the DAAs increase the risk of drug-drug interactions (DDIs).
Purpose Guided pharmaceutical interviews were conducted (i) to invite patients to provide feedback on the ADRs, to follow known DDIs, (ii) to encourage patients to communicate potential problems and to adapt pharmaceutical advice.

Materials and Methods The study was conducted between January and April 2012. Patient interviews on ADRs and DDIs were performed every month, during drug dispensing for outpatients by hospital pharmacists. They collected data based on questionnaires which included the documented adverse effects [1,2] and co-medications [3].

Results 56 questionnaires were completed with TVR patients and 65 with BOC patients. A total of 41 TVR and 62 BOC patients were examined for ADRs (data from the first month were excluded). Al patients had ADRs like those reported in the SPC (1,2). The most common ADRs were anaemia (52\%) and cutaneous manifestations (65\%), especially dry skin (44\%). Anaemia was more frequent in patients on BOC (56\% BOC/45\% TVR) but could be more severe with TVR: $55 \%$ of BOC patients and $29 \%$ of TVR patients were given erythropoietin and no BOC, but 3 TVR patients were transfused. Fatigue, rash, and pruritus were more frequent with TVR patients. Some ADRs were reported only by BOC patients: dysgeusia, alopecia and weight and appetite loss. Since DAAs are CYP 3A4 substrates and inhibitors, 58 potential interactions were identified and sometimes required close monitoring.

Conclusions Interviews enabled patients to talk about their ADRs and to express feelings on difficulties faced during their treatment. Hospital pharmacists gave them, in response, moral support and modified the advice they gave. They put patients' mind at rest about ADRs and raised patients' awareness of potential DDIs. Finally, the results on ADRs were reported to the health authorities in order to contribute to monitoring the risks related to these new drugs.

\section{References}

1. Summary of product characteristics (SPC) of telaprevir (Incivo). Available on the European Medicines Agency website: http://www ema.europa.eu

2. Summary of product characteristics (SPC) of boceprevir (Victrelis). Available on the European Medicines Agency website: http://www .ema.europa.eu

3. The hepatitis drug interactions website available at: http://www hep-druginteractions.org

No conflict of interest.

\section{GRP-042 COMPUTERIZED PHYSICIAN ORDER ENTRY IN THE GERIATRIC CENTER: COLLECTION AND ANALYSIS OF PRESCRIBING ERRORS MADE OVER A 5-MONTH PERIOD}

doi:10.1136/ejhpharm-2013-000276.042

C Cool, C Lebaudy, C Laborde, P Cestac. Teaching Hospital, Geriatric Center, Toulouse, France

Background Following the total computerization of prescriptions in the Geriatric Center over the past two years, the pharmaceutical team performs a pharmaceutical analysis for all the beds in the centre every day. Computerization is capable of reducing prescribing errors but it can generate some risks.

Purpose To collect, analyse and code the prescribing errors detected over a 5-month period, and to deduce the necessary actions to be taken in order to reduce the number and occurrence of errors.

Materials and Methods Research was carried out over the entire Geriatric Center: 314 beds (short, medium and long stays). Prescribing errors were collated daily and analysed via the computerised prescription software Disporao@. The proposed pharmaceutical interventions were communicated daily to the medical and care teams. The analysis and coding of the errors were carried out with 